\title{
In situ strain monitoring of fiber-reinforced polymers using embedded piezoresistive nanocomposites
}

\author{
Bryan R. Loyola $\cdot$ Valeria La Saponara • \\ Kenneth J. Loh
}

Received: 19 May 2010/Accepted: 15 July 2010/Published online: 31 July 2010

(C) The Author(s) 2010. This article is published with open access at Springerlink.com

\begin{abstract}
Fiber-reinforced polymer (FRP) structures and components are highly susceptible to damage due to delamination, matrix cracking, inter-laminar fracture, and debonding, all of which have potential to cause catastrophic structural failure. While numerous sensing technologies have been developed and embedded in FRP composites for monitoring strain, they serve as defects and can promote damage formation and propagation. Thus, in this study, an alternative technique is proposed for in situ strain monitoring of FRP composites via layer-by-layer multi-walled carbon nanotube-polyelectrolyte thin films deposited directly upon glass fiber weaves. To date, these carbon nanotube-based thin films have been validated for their piezoresistivity. The objective of this study is to characterize the strain sensing performance of different thickness thin films deposited on glass fiber weaves and embedded in FRP specimens using time-domain two-point probe resistance and frequency-domain electrical impedance spectroscopy (EIS) measurements. From the experimental thin film electromechanical response, a new method for fitting using a cubic smoothing spline is implemented and is compared to linear least-squares fitting. The results show that the cubic spline fit is better suited for capturing the strain sensitivities (or gage factors) of these thin films
\end{abstract}

\footnotetext{
B. R. Loyola · V. La Saponara ( $₫)$

Department of Mechanical and Aerospace Engineering, University of California, Davis, Davis, CA 95616, USA e-mail: vlasaponara@ucdavis.edu

URL: http://www.composites.ucdavis.edu
}

\section{K. J. Loh (ه)}

Department of Civil and Environmental Engineering,

University of California, Davis, Davis, CA 95616-5294, USA

e-mail: kjloh@ucdavis.edu

URL: http://www.nesst.engr.ucdavis.edu within the time- and frequency-domains along with the variation of strain sensitivity with applied strain. The bulk resistance response is described by the DC resistance measurements, whereas the EIS measurements provide insight of the inter-nanotube response.

\section{Introduction}

Fiber-reinforced polymer (FRP) composites fabricated from epoxy-infused carbon-, aramid-, and glass-fiber laminates are widely adopted for various engineering applications including aircraft, mechanical devices, naval structural components, wind turbine blades, and civil infrastructures (i.e., in particular, rehabilitation/retrofit of aging structures as well as for new construction). In fact, the aerospace industry has relied heavily on FRPs due to their low density, high strength-to-weight ratios, corrosion resistance, and conformability, all of which are more advantageous as compared to traditional structural materials such as aluminum. For instance, while the Boeing 767 airframe structure consists of $80 \%$ aluminum and $3 \%$ FRP (in 1982), the Boeing 787 that will begin service late 2010 is constructed from more than $50 \%$ composite materials [1]. On the other hand, FRPs have also been employed for bridge retrofit column jacketing and new construction; examples of real-world implementations include the Palazzo Elmi-Pandolfi historical building (Foligno, Italy) [2], the Interstate-80 State Street Bridge (Salt Lake City, UT, USA) [3], and the Kings Stormwater Channel Composite Bridge's FRP-based bridge deck (Riverside County, CA, USA) [4].

Despite the performance improvements offered by FRPs, these materials are highly susceptible to damage such as delamination, matrix cracking, inter-laminar 
fracture, and debonding, all of which can cause catastrophic structural failure [5, 6]. In fact, numerous sources are responsible for FRP damage including excessive loading, impact, fatigue, environmental-induced deterioration, material defects, and improper manufacturing. Moreover, FRP damage initiates at micron length scales and often occurs between laminae or at fiber/matrix interfaces, thereby being invisible to current visual inspection routines or even basic macro-scale sensor instrumentations such as strain gages, linear voltage displacement transducers, and accelerometers, among others. Nevertheless, the U.S. National Bridge Inspection Standards dictate that every bridge is to be visually inspected every 24 months for identifying significant structural damage before it jeopardizes operational safety and performance. However, damage that occurs between inspections can propagate and even escalate to cause brittle, explosive, and sudden catastrophic structural failure [7-9].

To date, a plethora of monitoring strategies and sensing technologies have been developed for structural health monitoring of aerospace systems [10,11], wind turbines [12], and civil structures [13], among others [14-19]. Some examples of these emerging sensing technologies include infrared thermography [20], shape-memory alloys [21], fiber optics [2], ultrasonics [22], and acoustic emissions [23]. For instance, fiber optics embedded in FRPs measure variations in the intensity or frequency content of reflected/refracted light traveling through a polymer-coated glass/silica waveguide in response to structural strain. Unfortunately, these optic fibers fail during instrumentation or operation, are easily broken, only measures damage along fiber lengths, and may promote stress concentrations within the composite parts [24]. On the other hand, ultrasonic and acoustic emissions rely on surface-bonded or embedded piezoelectric sensors/actuators to generate and detect guided waves for damage detection and defect localization [17]. Despite successful demonstrations of piezoelectric transducers for active sensing, they suffer from low signal-to-noise ratios due to electromagnetic interference, high computational demand, indirect damage detection, limited interrogation distance, and rely on complex algorithms or predefined damage metrics $[25,26]$. In addition, when embedded in FRPs similar to fiber optic systems, they can diminish structural performance and serve as defects that promote damage initiation.

In order to overcome the aforementioned limitations, many researchers have attempted to embed conductive nanomaterials within fiber-reinforced polymeric composites for encoding piezoresistivity (i.e., strain sensing capabilities). Most of these systems rely on embedding single- (SWNT) and multi-walled carbon nanotubes
(MWNT) [27] in FRP's epoxy matrices to try to take advantage of their superior physical, mechanical (e.g., SWNTs possess a Young's modulus, $E \approx 1.1 \mathrm{TPa}$ ), and electrical properties (i.e., near ballistic-transport electronic conductivity) [28]. For example, Böger et al. [5] have fabricated strain-sensitive carbon fiber-reinforced polymer (CFRP) materials by modifying the epoxy matrix with carbon black and nanotubes; the results are promising and have illustrated that the CFRP's electrical resistance changes in tandem with applied incremental tensile strains. A similar study has also been conducted by Thostenson and Chou [29] using $0.5 \mathrm{wt} \%$ MWNT-modified glass fiberreinforced polymers (GFRP). Unfortunately, these studies have relied on brute-force calendaring to force nanomaterial-epoxy mixtures through micron-sized orifices for dispersion and can lead to inadequate dispersion that limit composite piezoresistivity.

As opposed to dispersing nanomaterials within the FRP's epoxy matrix, the objective of this study is to deposit piezoresistive carbon nanotubes-based thin films directly onto glass fiber weaves and to investigate how film thickness affects its strain sensitivity. The nanocomposite-coated fiberglass is embedded in GFRP during composite fabrication for creating a self-sensing composite structure. It has been demonstrated in a separate study by Gao et al. [30] that thin films can be directly deposited onto glass fiber surfaces by dip-coating them in fully dispersed MWNT solutions and then drying the substrate. Although multifunctional sensing performance has been validated, the fabrication technique provides little control over nanomaterial assembly and the bulk film properties. Instead, in this study, a layer-by-layer (LbL) thin film fabrication methodology is employed for depositing piezoresistive MWNT-polyelectrolyte (PE) thin films onto the fiberglass weave; a total of four sets of films of different thicknesses are fabricated. Second, upon embedding this strain-sensitive fiberglass layer within GFRP samples, their strain-free electrical properties are characterized. Then, electromechanical testing is conducted for characterizing the strain sensing performance of nanocomposite-enhanced GFRPs. The GFRP samples are loaded in uni-axial tension while (1) the time-domain surface resistivity is measured, and (2) electrical impedance spectroscopy (EIS) is conducted to characterize the complex impedance response of the self-sensing GFRP system. Using the experimental EIS measurements, a simple equivalent circuit model is proposed for modeling the thin film impedance response to applied strains. Using the equivalent circuit model, individual circuit parameters are examined for their sensitivity to strain. Finally, this study concludes by comparing the experimental results and model fits from the time- and frequency-domain strain sensing results. 


\section{Experimental details}

\section{Substrate preparation}

Glass fiber weaves are employed as substrates for deposition and assembly of MWNT-PE thin films. Specimen preparation begins by cutting quasi-unidirectional glass fiber weaves (type 7715, Applied Vehicle Technology) into $20 \times 70 \mathrm{~mm}^{2}$ strips with the $0^{\circ}$ fibers parallel to the length of the specimens. These glass-fiber fabrics come pre-treated with a silane-based sizing agent to act as an adhesion promoter between the glass fibers and epoxy matrix, as is typical with most aerospace-grade fabrics. In addition, these sizing agents have been shown to adhere to poly(vinyl alcohol) (PVA, Sigma-Aldrich) well [16]. Since PVA is one of the main PE species used during film fabrication, its good adhesion to the substrate negates the need for harsh substrate chemical cleaning methods. Then, to prevent fraying and misalignment of the fibers, Super Glue Gel (Super Glue Corp.) is applied to the edges to create a semi-rigid framework that prevents folding of the fabric during the deposition process. The final step of the substrate preparation is to remove surface contaminants (such as dust) by rinsing the glass fabrics with $18 \mathrm{M} \Omega$ de-ionized water for $5 \mathrm{~min}$ and subsequently drying them with a stream of nitrogen gas for $5 \mathrm{~min}$.

\section{LbL thin film assembly}

The direct assembly of MWNT-PE thin films onto prepared glass fiber substrates is achieved via a LbL selfassembly process [31-34]. Previous studies conducted by Loh et al. [33, 34] have shown that homogeneous carbon nanotube-PE thin films with no phase segregation can be fabricated by depositing nanometer-thick monolayers of alternating charged PE and nanomaterials (Fig. 1). Since the glass fiber substrates possess an inherent negative electrical surface charge, the substrates are first immersed

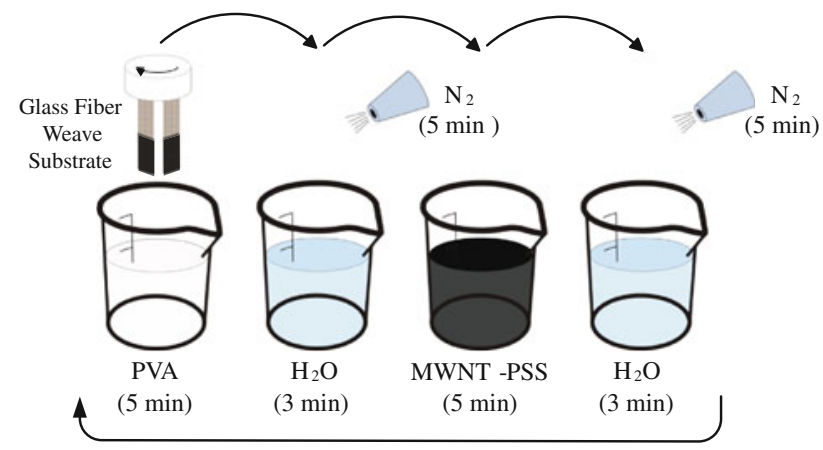

Fig. 1 A schematic illustrating the LbL deposition technique employed for fabricating (MWNT-PSS/PVA) $n$ thin films on glass fiber weaves in a polycationic $0.5 \mathrm{wt} \%$ PVA solution for $5 \mathrm{~min}$ to deposit the initial monolayer; here, adsorption of the initial PVA monolayer is due to covalent bonding between PVA's hydroxyl groups and the silane-coated substrate [35]. Upon deposition of the PVA monolayer, excess PE is removed by rinsing the substrate with $18 \mathrm{M} \Omega$ de-ionized water for 3 min followed by a 5.5-min drying phase with nitrogen gas. The LbL process continues by immersing the substrate (and the initial monolayer) in a negatively charged $1.0 \mathrm{mg} \mathrm{mL}^{-1} \mathrm{MWNT}$ (Cheap Tubes, less than $8 \mathrm{~nm}$ outer diameter) dispersed in $1.0 \mathrm{wt} \%$ poly(sodium 4-styrene sulfonate) (PSS, $M_{\mathrm{w}} \approx 1 \mathrm{M}$, Sigma-Aldrich) solution. Similar to previous studies by Loh et al. [33, 34], MWNTs are dispersed via steric stabilization by subjecting the MWNT-PSS solutions to $180 \mathrm{~min}$ of bath sonication (135 W, $42 \mathrm{kHz}$ ) followed by $30 \mathrm{~min}$ of high-energy tip sonication $(3.178 \mathrm{~mm}$ tip, $150 \mathrm{~W}, 20 \mathrm{kHz})$. It should be noted that MWNTs are used due to their intrinsic metallic nature and high electronic conductivities, whereas SWNTs can possess metallic or semi-conducting properties. Nevertheless, following the deposition of the MWNT-PSS polyanionic monolayer (and $3 \mathrm{~min}$ of rinsing and $5.5 \mathrm{~min}$ of drying), the MWNT-PSS monolayer adheres due to covalent bonding between PSS' sulfonate groups and PVA's hydroxyl groups [36]. The aforementioned procedure completes one bilayer of LbL thin film fabrication. Fabrication of robust, mechanically strong, and conductive thin films are achieved by repeating the deposition process numerous times (Fig. 1). These nanocomposites are denoted in this article as (MWNT-PSS/PVA) ${ }_{n}$, where $n$ refers to the number of bilayers.

Upon film fabrication, scanning electron microscopy (SEM) is employed for imaging the surface and crosssectional morphologies of the nanocomposites. From Fig. 2, the MWNTs form a dense percolated structure that is crucial for enabling electrical conductivity and high mechanical performance. It can also be seen from Fig. 2 that only individual and small bundles of MWNTS are deposited during fabrication. For this study, films of four different thicknesses (i.e., 29, 50, 100, and 150 bilayers) are fabricated and directly deposited onto fiber weaves for electromechanical testing.

\section{Fabrication of nanocomposite-enhanced GFRPs}

Once the thin films are deposited on the glass fiber substrates, each unique sample is cut into narrow specimens that contain three rovings (a bundle of fibers) in the $0^{\circ}$ direction to minimize transverse strain due to Poisson's effect. The resulting thin film-fiber samples are approximately $3 \mathrm{~mm}$ wide and $70 \mathrm{~mm}$ long. However, it should be noted that the thin films are only deposited in a region up to $30 \mathrm{~mm}$ from the bottom of the glass weave. For this reason, 

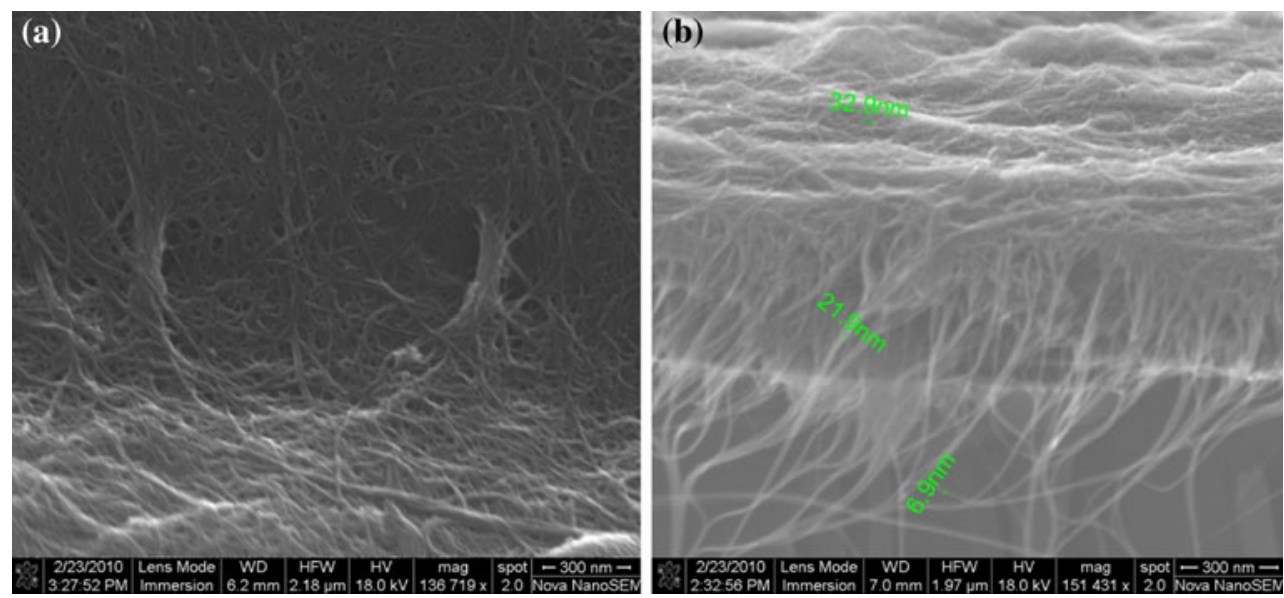

Fig. 2 a Surface and $\mathbf{b}$ cross-sectional SEM images of a carbon nanotube-based thin film showing the fully percolated and random oriented nanotube network. The images suggest that only individual or small bundles of carbon nanotubes are deposited during LbL assembly

electrodes are placed 10 and $25 \mathrm{~mm}$ along the length and from the bottom of the specimen to create a $15-\mathrm{mm}$ electrical gage length. For each specimen, the electrodes are formed by using conductive silver paint (Ted Pella) for attaching 28 AWG single-stranded wire to each film's surface. After electrode attachment, the samples are infused with a two-part epoxy (125/237 Proset laminating epoxy system) to fabricate the GFRP composites; the epoxy embeds the nanocomposite within the GFRP and secures the conductive electrodes. Once infused, the samples are cured per manufacturer specification for $15 \mathrm{~h}$ at $27^{\circ} \mathrm{C}$ followed by $8 \mathrm{~h}$ at $82{ }^{\circ} \mathrm{C}$.

Since these specimens will undergo electromechanical tensile testing, GFRP tabs are applied to the two ends of the GFRP specimens to prevent grip damage. Here, the $19 \times 19 \mathrm{~mm}^{2}$ tabs are cut and bonded to the nanocomposite-enhanced GFRP specimens using Hysol 907 adhesive. The tabs are placed in a manner to also cover the end of the two electrodes for providing an added layer of protection for the electrodes. Once the tabs are adhered, the adhesive is cured at $60{ }^{\circ} \mathrm{C}$ for $2 \mathrm{~h}$. The finally prepared specimen is shown in Fig. 3.

\section{Electromechanical tensile testing}

Validation of the electromechanical strain sensing performance of the nanocomposite-enhanced GFRP specimens is obtained by commanding a TestResources 150R load frame to apply uni-axial tensile loads to the as-prepared specimens. The displacement profile executed by the load frame is shown in Fig. 4. It can be observed from Fig. 4 that the displacement profile includes pauses at discrete strain states to permit electrical interrogation of the embedded nanocomposite. For this study, the load frame's displacement rate is set to $1 \mathrm{~mm} \mathrm{~min}^{-1}$, which is a
Fig. 3 The final manufactured nanocomposite-embedded GFRP specimen

compromise between the ASTM D3039 standard and the load frame's sampling rate capabilities [37]. In addition, from Fig. 4, it can also be seen that two different profiles are included, namely (1) a low-strain regime from 0 to $10000 \mu \varepsilon$ and (2) a high-strain regime from 15000 to $\sim 100000 \mu \varepsilon$ or until sample failure. First, during the lower strain regime, the load frame is paused for $60 \mathrm{~s}$ at every $1000 \mu \varepsilon$ to allow time for conducting electrical measurements. The measurements here are designed to capture the embedded nanocomposite's electrical response within the elastic region of the GFRP samples. Secondly, for the highstrain region, the objective is to capture damage in the specimens due to transverse cracking or other damage modes; in this high-strain regime, 60 s pauses are executed 


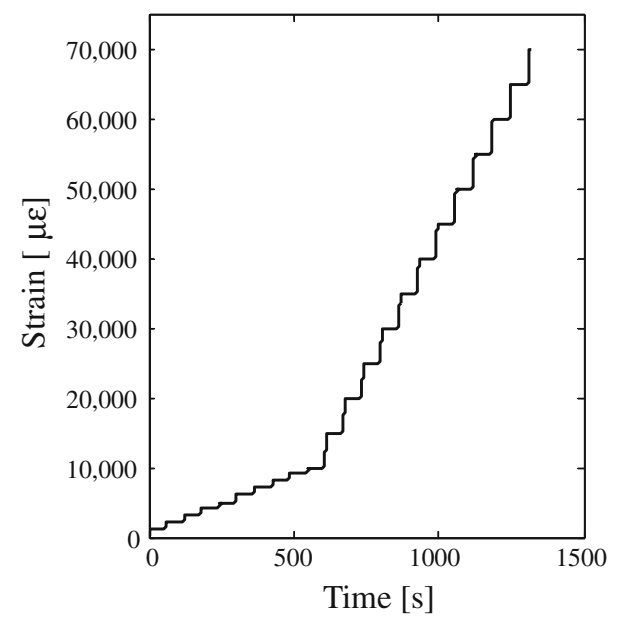

Fig. 4 The time-displacement profile that is executed by the TestResources 150R load frame for tensile testing

every $5000 \mu \varepsilon$ increments. Regardless, the displacement rate of the load frame remains constant during tensile testing.

Time- and frequency-domain electromechanical measurements

As mentioned earlier, during each applied strain state and when the load frame holds its load and displacement, the embedded thin film's electrical response to applied strain is queried via two methods, namely, the time-domain surface resistivity measurements and frequency-domain EIS. First, the time-domain direct current (DC) resistance is measured using an Agilent 34401A digital multimeter via a two-point probe electrode setup. Immediately following the surface resistance measurement, EIS is conducted to characterize the nanocomposite's complex impedance response to applied strain. While time-domain resistance measurements allow for characterization of the average/bulk electrical properties of the embedded films, EIS investigates how the thin films respond to electrical stimuli of different alternating current (AC) frequencies [38].

In short, EIS is performed using a frequency response analyzer such as an Agilent 4294A precision impedance analyzer. The analyzer applies two monochromatic AC currents $90^{\circ}$ out-of-phase from one another and measures the corresponding voltage magnitude and phase response. With the input $\mathrm{AC}$ current and output voltage (i.e., magnitude and phase) information, the thin film's impedance $\left(Z(\omega)=Z^{\prime}+j Z^{\prime \prime}\right)$ at the applied AC signal's frequency, $\omega$, can be calculated as shown in Eq. 1

$$
\begin{aligned}
Z(\omega) & =V(\omega) / i(\omega)=Z^{\prime}(\omega)+j Z^{\prime \prime}(\omega)=|Z(\omega)| e^{j \varphi(\omega)} \\
& =|Z(\omega)| \angle \varphi(\omega)
\end{aligned}
$$

However, instead of determining the film's complex impedance response at a fixed frequency, the Agilent 4294A impedance analyzer can be commanded to input AC signals spanning a wide range of different frequencies. By doing so, one can also use the collected complex impedance spectrum for modeling the thin film as an equivalent circuit consisting of resistors, capacitors, and inductors. This model not only captures the bulk response of the thin films (similar to the time-domain response), but the circuit model can also illuminate electron and hole transport phenomena at nanometer length scales. In this study, the impedance analyzer is set to input $500 \mathrm{mV}$ AC signals of frequencies ranging from $40 \mathrm{~Hz}$ to $1 \mathrm{MHz}$. For every $\mathrm{AC}$ signal frequency, the corresponding complex impedance response is automatically recorded for capturing the EIS spectrum at each applied strain state.

\section{Results and discussion}

Frequency-domain equivalent circuit model fitting

In this study, time-domain (i.e., DC resistance) and frequency-domain (i.e., EIS) measurements are taken to characterize thin film electrical properties and piezoresistive response. Unlike resistance measurements, frequencydomain electrical impedance spectroscopic data must undergo post-processing to extract pertinent information. In fact, the EIS spectrum can be fitted to an equivalent circuit model for characterizing thin film resistive, capacitive, and/ or inductive electronic behavior [38]. To illustrate this point, a representative EIS response is displayed in a Nyquist plot, or a plot with the real component of impedance on the $x$-axis and the negative imaginary component of impedance on the $y$-axis as shown in Fig. 5a. To fit the nanocomposite's as-measured EIS response, the equivalent circuit model shown in Fig. $5 b$ is proposed; this type of parallel resistor-capacitor circuit model has also been used to model the response of zirconia-yttria solid electrolytes [39]. From Fig. 5b, the parallel resistor $\left(R_{\mathrm{p}}\right)$ and parallel capacitor $\left(C_{\mathrm{p}}\right)$ represent the respective resistance and capacitance of the inter-granular boundaries of the metallic grains, whereas $R_{\mathrm{S}}$ models the resistance within the grains themselves [39]. Thus, this model is analogous to the nanotube-based film, where $R_{\mathrm{p}}$ and $C_{\mathrm{p}}$ model the internanotube junctions, whereas $R_{\mathrm{S}}$ represents the bulk resistance of the film. The equivalent complex impedance of the proposed circuit model is also derived as shown in Eq. 2

$$
\begin{aligned}
Z(\omega) & =Z^{\prime}(\omega)+i Z^{\prime \prime}(\omega) \\
& =\left(R_{\mathrm{s}}+\frac{1 / R_{\mathrm{p}}}{\left(1 / R_{\mathrm{p}}\right)^{2}+\omega^{2} C_{\mathrm{p}}^{2}}\right)-i\left(\frac{\omega C_{\mathrm{p}}}{\left(1 / R_{\mathrm{p}}\right)^{2}+\omega^{2} C_{\mathrm{p}}^{2}}\right)
\end{aligned}
$$



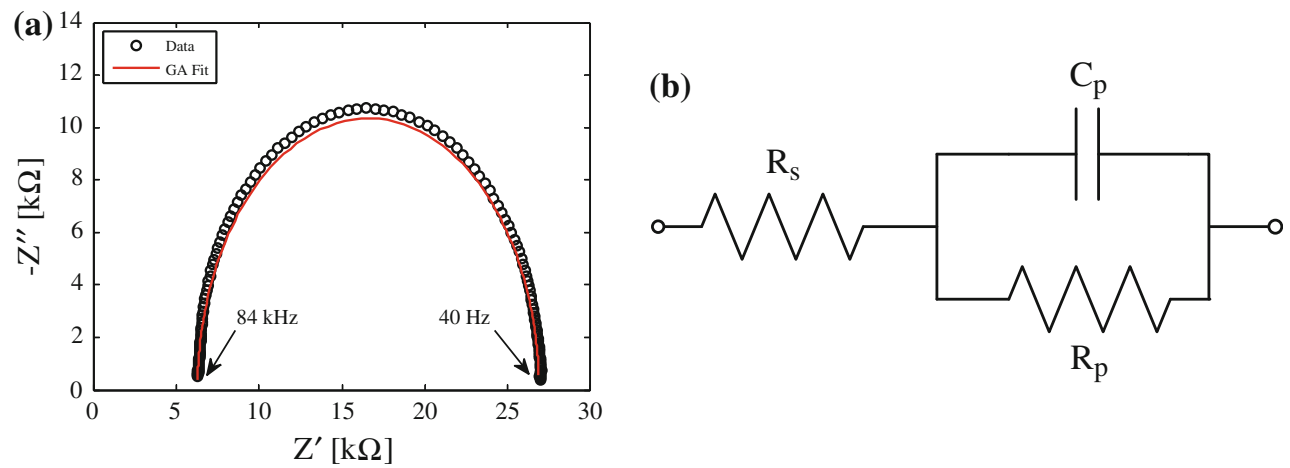

Fig. 5 a A representative Nyquist plot of an (MWNT-PSS/PVA) $)_{50}$ thin film's complex impedance response, and b the proposed equivalent circuit model employed for GA fitting

To fit the collected impedance data to this non-linear model (Fig. 5b and Eq. 2), a genetic algorithm (GA) is utilized using MATLAB's GA toolbox. Here, the GA is used to determine $R$ and $C$ for each impedance spectrum, whereas $R$ caln be simply obtained from the left $x$-intercept of the impedance arc. The fitness function with which the GA will minimize is based on the imaginary component of the complex impedance (Eq. 2) and equals the area between the experimental data and the model with the proposed circuit element values. For simplicity, the trapezoidal method is employed for computing the fitness function, but this technique is also accurate due to the large number of impedance data points collected. Although the impedance spectrum is taken over a range of logarithmically spaced frequencies from $40 \mathrm{~Hz}$ to $1 \mathrm{MHz}$, only the impedance corresponding to frequencies less than $84 \mathrm{kHz}$ are needed to obtain an accurate fit. Thus, the GA fitting is only conducted using impedance data corresponding to frequencies from $40 \mathrm{~Hz}$ to $84 \mathrm{kHz}$ to minimize computational time. The representative fit is also overlaid in Fig. 5a for validation.

Strain-free nanocomposite electrical properties: effects of epoxy infusion

Prior to tensile testing, each film's DC resistance response is measured before and after the infusion of epoxy. The first objective is to explore the effects of epoxy on the nanocomposite's inherent electrical properties. Since four different film thicknesses are explored, instead of comparing the absolute resistance values, the percent change in resistance before and after epoxy infusion is calculated and tabulated in the first row of Table 1 . As can be seen from the first row of Table 1, the infusion of epoxy causes the bulk resistance of the films to increase, as has also been observed by other researchers [40, 41]. In particular, the
Table 1 Average percent change of thin film electrical properties due to infusion of epoxy

\begin{tabular}{lcrlr}
\hline $\begin{array}{l}\text { Circuit } \\
\text { element }\end{array}$ & 29 Bilayers & 50 Bilayers & $\begin{array}{l}100 \\
\text { Bilayers }\end{array}$ & \multicolumn{1}{l}{$\begin{array}{l}150 \\
\text { Bilayers }\end{array}$} \\
\hline$R_{\mathrm{DC}}$ & $148.7 \pm 24.2$ & $67.5 \pm 2.0$ & $40.1 \pm 1.2$ & $28.3 \pm 0.9$ \\
$\quad\left(\% \Delta R_{\mathrm{DC}}\right)$ & & & & \\
$R_{\mathrm{s}}\left(\% \Delta R_{\mathrm{s}}\right)$ & $148.4 \pm 22.3$ & $61.6 \pm 1.7$ & $31.8 \pm 0.5$ & $22.2 \pm 0.5$ \\
$R_{\mathrm{p}}\left(\% \Delta R_{\mathrm{p}}\right)$ & $-16.1 \pm 2.9$ & $10.9 \pm 0.5$ & $6.1 \pm 0.2$ & $4.6 \pm 0.0$ \\
$C_{\mathrm{p}}\left(\% \Delta C_{\mathrm{p}}\right)$ & $31.1 \pm 11.5$ & $-0.1 \pm 0.1$ & $-0.9 \pm 0.1$ & $-0.3 \pm 0.1$ \\
\hline
\end{tabular}

increase in resistance is inversely related to film thickness; 29 bilayer films increase their resistance by an average of $148.7 \%$, whereas the 150 bilayer sample set increases only $28.3 \%$ (Table 1). Pham et al. [40] have also reported similar findings during their study of infusing buckypapers of various thicknesses with polycarbonate.

Using the EIS measurements and upon equivalent circuit model fitting, the average percent change in equivalent circuit element values is also tabulated in Table 1. It can be seen from Table 1 that the results for $R_{\mathrm{s}}$ correspond with that of $R_{\mathrm{DC}}$, thereby further demonstrating that $R_{\mathrm{S}}$ models the bulk film nanocomposite electrical properties. As mentioned earlier, the DC resistance of nanocomposites increases after the infusion of epoxy, and the average percent increase in resistance decreases with increasing number of bilayers (or increasing film thickness). For the parallel equivalent circuit elements, the same trends seem to apply to $R_{\mathrm{p}}$ as well, with the exception of the 29 bilayer sample set which demonstrates a decrease in resistance after infusion. For the parallel capacitor circuit element, $C_{\mathrm{p}}$ for the 29 bilayer sample set increases by $31 \%$ after infusion, but otherwise, infusion has negligible effects on thin film parallel capacitance. The effects governing the changes in the parallel circuit elements due to epoxy infusion is still not well understood and will be a future focus of investigation. 
Table 2 Mean measured DC resistance values, corresponding mean electrical gage lengths, and calculated DC resistivities for each unique thin film sample set

\begin{tabular}{llll}
\hline Film thickness & $\begin{array}{l}\text { Mean } R_{\mathrm{DC}} \\
(\mathrm{k} \Omega)\end{array}$ & $\begin{array}{l}\text { Mean gage } \\
\text { length }(\mathrm{cm})\end{array}$ & $\begin{array}{l}\text { Mean resistivity } \\
\left(\mathrm{k} \Omega \mathrm{cm}^{-1}\right)\end{array}$ \\
\hline 29 Bilayers & $7.09 \pm 0.41$ & $1.467 \pm 0.046$ & $4.91 \pm 0.26$ \\
50 Bilayers & $6.71 \pm 0.60$ & $1.684 \pm 0.051$ & $3.93 \pm 0.36$ \\
100 Bilayers & $2.94 \pm 0.37$ & $1.492 \pm 0.040$ & $1.65 \pm 0.12$ \\
150 Bilayers & $1.51 \pm 0.52$ & $1.586 \pm 0.059$ & $0.56 \pm 0.02$ \\
\hline
\end{tabular}

Strain-free nanocomposite electrical properties: effects of film thickness

The second objective in this study is to characterize the strain-free inherent nanocomposite electrical properties and the effects of film thickness (or number of bilayers). However, instead of only comparing average film resistance measurements, the DC electrical resistance of each specimen can also be normalized with the film's geometry by computing its surface resistivity, $\rho$, as shown in Eq. 3

$\rho=\frac{R_{\mathrm{DC}}}{l}$

where $R_{\mathrm{DC}}$ is the two-point probe DC resistance measurement as before, and $l$ is the gage length. The average DC resistance, gage length, and resistivity values for the different sample sets are presented in Table 2. It can be seen from Table 2 that the average film DC resistance and surface resistivity decreases in tandem with increasing thickness. In addition, for the specimens employed in this study, the nanocomposite DC resistances are in the 1 to $10 \mathrm{k} \Omega$ range (Table 2).

The circuit element values for the unloaded or strainfree specimens of varying thicknesses are plotted in Fig. 6 and listed in Table 3. In the case of the DC resistance $\left(R_{\mathrm{DC}}\right)$ and series resistor $\left(R_{\mathrm{S}}\right.$ of the equivalent circuit model in Fig. 5b), resistance is inversely related to film thickness due to the increase in the number of nanotubes in the film (Fig. 6) as have also been observed in other studies [34, 42]. As mentioned earlier, $1.0 \mathrm{mg} \mathrm{mL}^{-1}$ MWNT in $1.0 \mathrm{wt} \%$ PSS solutions are employed for LbL nanocomposite fabrication. While percolation theory can be used to determine the lowest volume percentage of conductive fillers required for the films to be electrically conductive (i.e., by exceeding the percolation threshold) [43-46], the MWNT concentrations used for film fabrication creates a three-dimensional quasi-homogeneous network of MWNTs. These networks can be observed in the surface and cross-sectional SEM images in Fig. 2 and has also been confirmed in previous studies [33, 34]. By exceeding the percolation threshold, the drop in resistance with increasing thickness can be explained by the growing number of conductive pathways through which electrical current can travel and is consistent with percolation

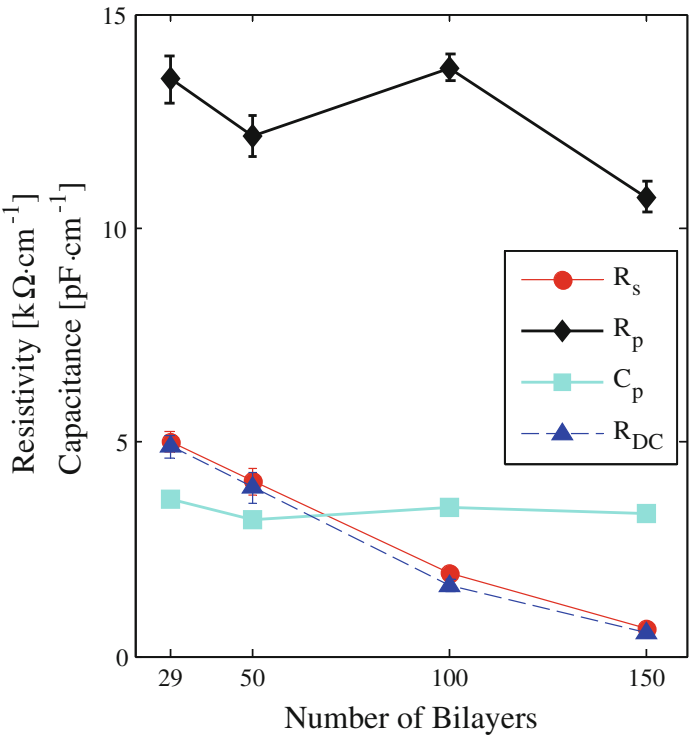

Fig. 6 The average initial values of the EIS equivalent circuit elements and DC surface resistivities for each thin film sample set with 29, 50, 100, and 150 bilayers. The mean error is also plotted as shown in the error bars

theory. In contrast, the initial values of the circuit elements representing the inter-nanotube connections should not be sensitive to the thickness of the film, and this is clearly evident by Fig. 6 .

\section{Strain sensing performance characterization}

As mentioned in previous sections and shown in Fig. 4, the load frame is paused at fixed strain intervals during tensile testing to allow for time- and frequency-domain electrical measurements. First, we verify that the EIS measurements are indeed strain sensitive. Select impedance arcs recorded at specified strain values are overlaid in the Nyquist plot in Fig. 7a. From Fig. 7a it can be clearly seen that the height of the arc is decreasing while simultaneously shifting to the right in tandem with increasing applied strains. To better understand this piezoresistive response, each impedance spectrum is fit to the equivalent circuit model of Fig. $5 \mathrm{~b}$ using the same GA technique. Once the fits are complete, the equivalent circuit element values (i.e., $R_{\mathrm{s}}, R_{\mathrm{p}}$, and $C_{\mathrm{p}}$ ), 
Table 3 Average initial or strain-free DC resistance and EIS equivalent circuit element values for each thin film sample set

\begin{tabular}{lrrrr}
\hline Circuit element & \multicolumn{1}{c}{ 29 Bilayers } & 50 Bilayers & 100 Bilayers & 150 Bilayers \\
\hline$R_{\mathrm{DC}}\left(\mathrm{k} \Omega \mathrm{cm}^{-1}\right)$ & $4.91 \pm 0.26$ & $3.93 \pm 0.36$ & $1.65 \pm 0.12$ & $0.56 \pm 0.02$ \\
$R_{\mathrm{s}}\left(\mathrm{k} \Omega \mathrm{cm}^{-1}\right)$ & $5.00 \pm 0.24$ & $4.08 \pm 0.32$ & $1.92 \pm 0.12$ & $0.65 \pm 0.02$ \\
$R_{\mathrm{p}}\left(\mathrm{k} \Omega \mathrm{cm}^{-1}\right)$ & $13.48 \pm 0.56$ & $12.13 \pm 1.48$ & $13.75 \pm 0.32$ & $10.72 \pm 0.36$ \\
$C_{\mathrm{p}}\left(\mathrm{pF} \mathrm{cm} \mathrm{cm}^{-1}\right)$ & $3.67 \pm 0.11$ & $3.21 \pm 0.12$ & $3.48 \pm 0.09$ & $3.34 \pm 0.13$ \\
\hline
\end{tabular}

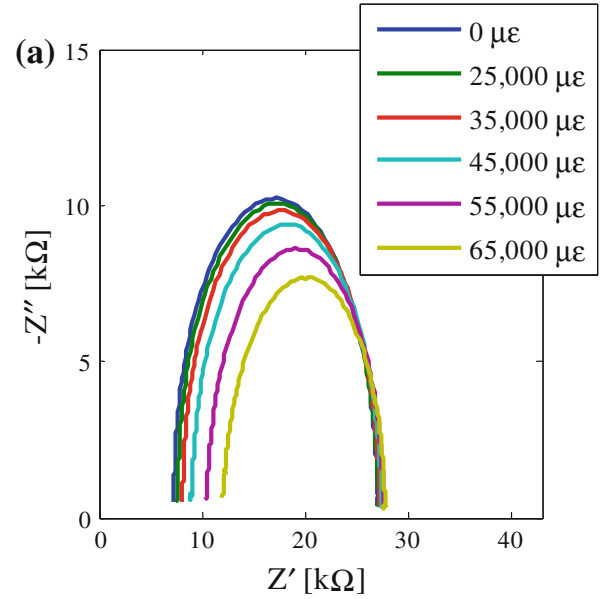

Fig. 7 a An overlay of an (MWNT-PSS/PVA) $)_{50}$ thin film's complex impedance response at different applied strain states. As the strain increases, the complex impedance term becomes less negative, while the left real component of impedance increases. b Equivalent circuit

along with the DC resistance measurements, are plotted versus strain as shown in Fig. 7b. The following section discusses the most appropriate method with which to fit the behavior of the nanocomposites' strain sensitivities.

\section{Circuit element strain response fitting}

The typical method for reporting the strain sensitivity of a piezoresistive material is to calculate the strain sensitivity or gage factor $(S)$ (i.e., the normalized change in resistance (or capacitance) divided by the corresponding change in applied strain):

$S=\frac{\Delta R / R_{0}}{\Delta \varepsilon}$

In the case of numerous piezoresistive materials presently investigated for strain sensing and structural monitoring applications, most of them often demonstrate linear strain sensitivities with a few demonstrating a bilinear sensitivity in dynamic or monotonic load tests [33, 34, 42, 47-54]. In these studies, the researchers typically report the gage factor in these linear regions using a least-squares linear fit to identify the slope (thus the gage factor). The results shown in Fig. $7 \mathrm{~b}$ are clearly at least bilinear, with several

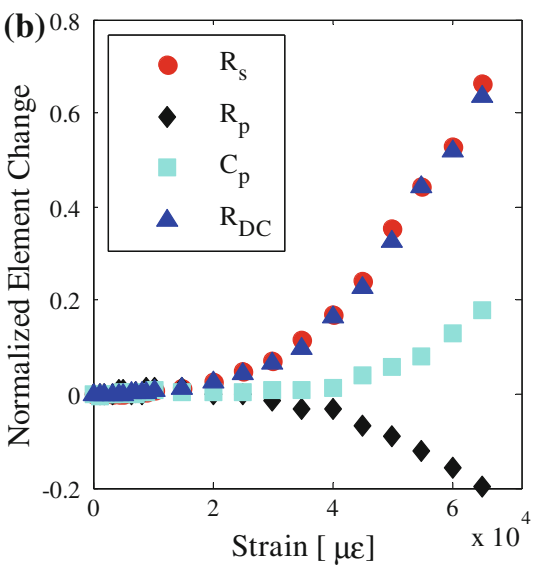

model fitting for the corresponding film is conducted to extract each circuit element's value during each applied strain state, and their normalized change is plotted as a function of applied strain. It should be noted that $R_{\mathrm{p}}$ exhibits a negative strain sensitivity

datasets demonstrating trilinear relationships (particularly for thin films of smaller thicknesses). Since the objective of this research is to characterize the strain sensing performance of (MWNT-PSS/PVA) ${ }_{n}$ thin films, an accurate characterization of the strain sensitivity over the entire sensor's dynamic range is desired.

For this study, three methods of model fitting are applied to the experimental data for extracting the films' corresponding strain sensitivities. The first technique utilizes MATLAB's non-linear solver to apply a multi-linear fit to the data. Given the number of linear regions to fit and an initial estimate for the transition points, the solver optimizes the fit by determining the optimum slopes and transition points corresponding to each linear segment. The second fitting method continues to utilize the multi-linear fit, but takes advantage of locally weighted scatter plot smoothing (LOWESS). The LOWESS smoothing algorithm uses a tri-cubic weighting function (Eq. 5) to smooth the data using only the data points within the specified span (in this case $30 \%$ of the data) while performing a local linear fit with least-squares. The span chosen here is based on the approximate size of the linear regions from the multi-linear fits (i.e., the first-method). In the method that MATLAB uses (Eq. 5), $\mathrm{d}(x)$ is the distance along the 
abscissa from the point being smoothed $(x)$ and the point for which the weighting function, $w\left(x_{i}\right)$, is being calculated [55]

$w\left(x_{i}\right)=\left(1-\left(\frac{x-x_{i}}{\mathrm{~d}\left(x_{i}\right)}\right)^{3}\right)^{3}$

This smoothing method has two distinct advantages over other methods such as a moving-average. The LOWESS fit is designed to locally smooth the data to a first or secondorder polynomial fit while needing only a span to be specified. Since the largest disadvantage is that the algorithm is unable to return a regression function, the data must be fit using the multi-linear fitting scheme [56]. As the intent of the smoothing algorithm is to allow for a better multi-linear fit, this disadvantage is mitigated. Finally, the third fitting method involves fitting the data with a cubic smoothing-spline, or a piece-wise cubic fit to the data, with the smoothing based on a smoothing parameter. The smoothing parameter can be specified between 0 and 1, with 0 consisting of a least-square's linear fit, and 1 giving a spline that matches the position of each data point perfectly. In this case, the smoothest fit is desired, and the smoothing parameter of 0.9999999 is adopted. ${ }^{1}$ Based on fitting the data with several smoothing parameter values in this regime, it has been found that this smoothing parameter captured very well the data without causing over-smoothing, particularly in the case of the $R_{\mathrm{s}}$ and $R_{\mathrm{DC}}$ trends (i.e., over-smoothing would appear with oscillations around the apparent trend in the data).

As a way to compare the bulk piezoresistive behavior from this study to that of others, the average low-strain (0 to $10000 \mu \varepsilon$ ) bulk resistance gage factors are determined for each circuit elements $R_{\mathrm{DC}}$ and $R_{\mathrm{S}}$ for films of all four thicknesses using the multi-linear fit. By averaging over this region, the strain response is determined in the same strain regime comparable to other studies. These averaged values, as listed in Table 4, clearly show that the bulk resistance strain sensitivity is inversely proportional to film thickness.

The strain sensitivity data for the three EIS equivalent circuit parameters and the DC resistance for a (MWNTPSS/PVA) ${ }_{29}$ nanocomposite are plotted in Fig. 8. Figure 8a-c plots the experimental data for $R_{\mathrm{DC}}, R_{\mathrm{S}}, \mathrm{R}_{\mathrm{p}}$, and $C_{\mathrm{p}}$ and their corresponding multi-linear, LOWESS, and spline fit, respectively. As indicated by these plots, all of the fitting methods accurately fit the trend of the circuit elements and $R_{\mathrm{DC}}$. To quantitatively determine the goodness-of-fit, the root-mean-square (RMS) of the residuals

\footnotetext{
1 Although seemingly very close to 1 , this parameter is very close to the value of the sensitivity range of the smoothing parameter as defined by the MATLAB help file, which specifies a smoothing parameter of 0.99999999 or eight $9 \mathrm{~s}$ instead of seven [55].
}

Table 4 The average low-strain (i.e., 0 to $10000 \mu \varepsilon$ ) bulk resistance strain sensitivity determined using the multi-linear fitting method for nanocomposite sample sets of $29,50,100$, and 150 bilayers

\begin{tabular}{lll}
\hline $\begin{array}{l}\text { Number } \\
\text { of bilayers }\end{array}$ & $\begin{array}{l}R_{\mathrm{DC}} \text { strain } \\
\text { sensitivity }\end{array}$ & $\begin{array}{l}R_{\mathrm{s}} \text { strain } \\
\text { sensitivity }\end{array}$ \\
\hline 29 & $1.37 \pm 0.09$ & $1.01 \pm 0.00$ \\
50 & $1.06 \pm 0.11$ & $1.18 \pm 0.10$ \\
100 & $0.86 \pm 0.13$ & $0.79 \pm 0.07$ \\
150 & $0.55 \pm 0.03$ & $0.54 \pm 0.02$ \\
\hline
\end{tabular}

are calculated for each fit and examined. The results of the fit for the (MWNT-PSS/PVA) 29 sample set shown in Fig. 8 are also transcribed in Table 5. From Table 5, it can be seen that the spline performs the best at fitting the strain sensitivity data due to the lowest RMS residuals. In fact, this dataset represents the general goodness-of-fit across all of the specimens and sample sets. Typically, the multilinear and LOWESS fits are closely matched, and the spline fits possess RMS residuals that are at least $10 \%$ lower. In addition to the overall better goodness-of-fit, the method of spline fitting has the distinct advantage of not requiring a specific basis function with which to fit to but returns the regression function in terms of piece-wise cubic functions from which the slope and gage factor can be calculated. For these reasons, the strain sensitivities will be analyzed herein using the method of spline smoothing.

Bulk film resistance strain sensitivity analysis

Once the strain response of the EIS equivalent circuit elements and DC resistance are fitted via the cubic smoothing splines, the average strain responses of the four sets of films of different thicknesses (i.e., 29, 50, 100, and 150 bilayers) are determined. As all of the electrical measurements are taken for the same set of strain values, the normalized change of the circuit elements and their gage factors at these specified strain points are averaged, and the error of the mean is determined. Since spline fits are returned as a piece-wise continuous function across the region of interest, the gage factors at each applied strain state are calculated. The mean gage factors are presented in Fig. 9, with each circuit element plotted separately on each row. The left-hand-side plots of Fig. 9 only show the 0 to $10000 \mu \varepsilon$ region and are a magnification of the right-handside plots of Fig. 9.

From the results of spline fitting, $R_{\mathrm{S}}$ and $R_{\mathrm{DC}}$ have nearly identical sensitivities to strain. In the low-strain region $(0$ to $10000 \mu \varepsilon$ ), the gage factors tend to stay constant across this range and decrease in value as the number of bilayers increases (i.e., with increasing film thicknesses). It should be noted that the (MWNT-PSS/PVA) $)_{50}$ films have a higher average strain sensitivity than the films with 29 bilayers. It 

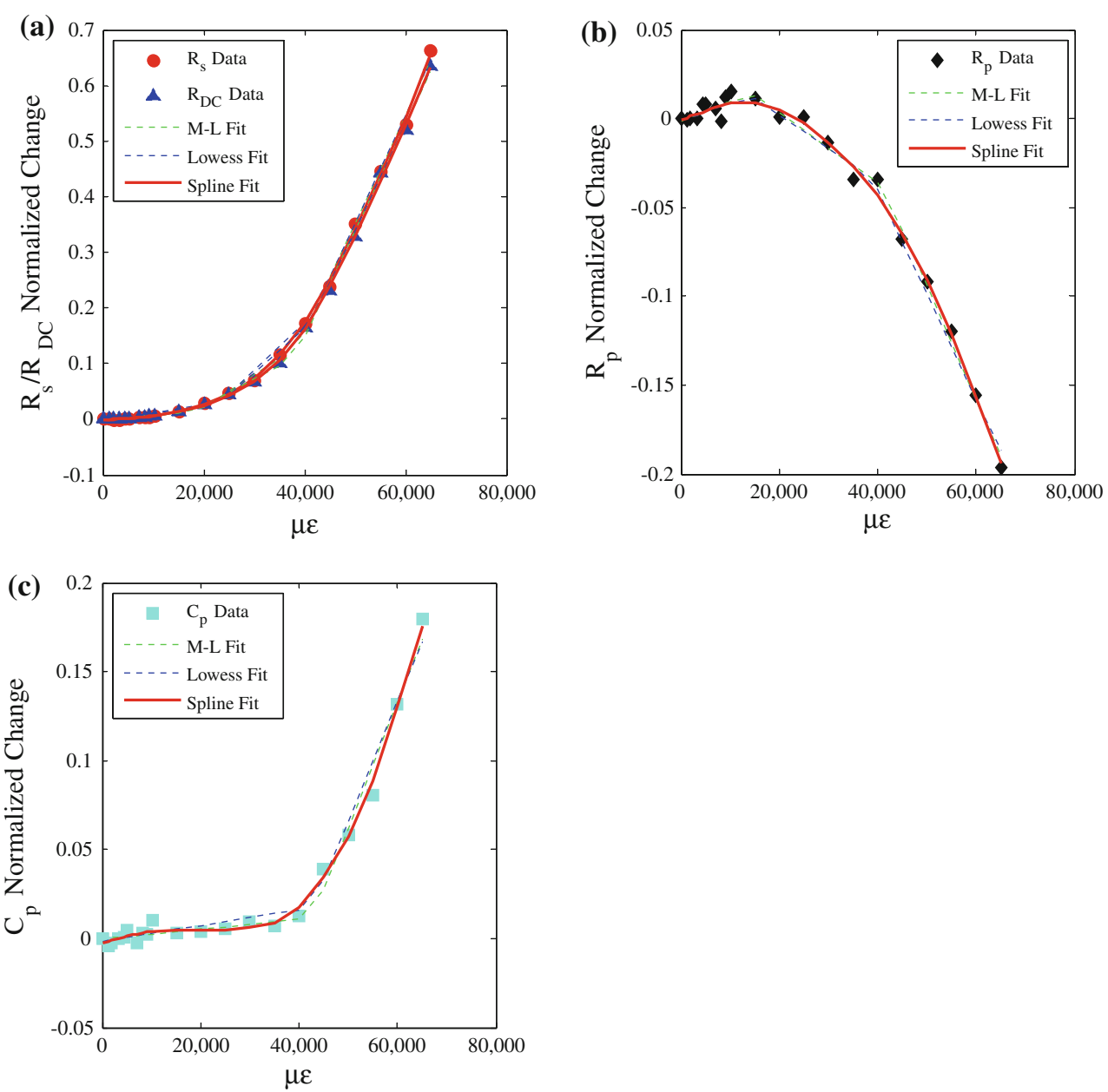

Fig. 8 The (MWNT-PSS/PVA) 29 thin film's a bulk resistance (i.e., $R_{\mathrm{DC}}$ and $\left.R_{\mathrm{s}}\right)$, b inter-nanotube resistance $\left(R_{\mathrm{p}}\right)$, and $\mathbf{c}$ inter-nanotube capacitance $\left(C_{\mathrm{p}}\right)$ response to applied strain

Table 5 The RMS residuals of the three fitting methods for a representative (MWNT-PSS/PVA) 29 thin film

\begin{tabular}{lllll}
\hline & $R_{\mathrm{DC}}$ & $R_{\mathrm{s}}$ & $R_{\mathrm{p}}$ & $C_{\mathrm{p}}$ \\
\hline Multi-linear & 0.0014 & 0.0017 & 0.0010 & 0.0013 \\
LOWESS & 0.0018 & 0.0011 & 0.0014 & 0.0018 \\
Spline & 0.0009 & 0.0010 & 0.0009 & 0.0007 \\
\hline
\end{tabular}

is presumed that films of smaller thicknesses are less homogeneous than those with higher thicknesses to cause this discrepancy. In the case of depositing thin films upon a substrate as uneven as fiber bundles, it is also hypothesized that this effect is somewhat exaggerated.

Above this strain regime (i.e., at applied strains greater than $10000 \mu \varepsilon$ ), the clear separation between the relationship of the strain sensitivities and film thickness disappears. For all the different sets of films, there is an average linear increase in strain sensitivity with applied strain, but the results are also associated with large amounts of noise as indicated by the large error bars. However, for both circuit elements $R_{\mathrm{DC}}$ and $R_{\mathrm{s}}$, the (MWNT-PSS/PVA) ${ }_{29}$ film set exhibits the highest gage factors in the high-strain region. It is in this region that other research groups have determined a non-linear change in resistance, but in fact, this study shows a linear change in gage factor. There may be a second-order change in resistance potentially due to microcracking within the matrix, but additional work is required to confirm this hypothesis. Some researchers have created damage indices to quantify these changes [5, 29, 57].

Inter-nanotube strain sensitivity analysis

The strain response of the equivalent circuit elements (i.e., $R_{\mathrm{p}}$ and $C_{\mathrm{p}}$ ) modeling the inter-nanotube junctions behaves very differently than that of the aforementioned bulk nanocomposite resistive properties. In the low-strain region, the strain sensitivity is very small and non-linear for $R_{\mathrm{p}}$ and $C_{\mathrm{p}}$, and in the case of certain film thicknesses can even change sign. This non-linear behavior may be caused by the carbon nanotubes reorganizing and aligning 
Fig. 9 Gage factors for each circuit element as a function of strain for the different (MWNTPSS/PVA $)_{n}$ films are shown. The plot on the left of each row is a magnification of the lowstrain region (i.e., 0 to 10000 $\mu \varepsilon)$ for that corresponding circuit element's response to applied strain. The plots on the right show the strain sensitivity results for the entire range of applied strains
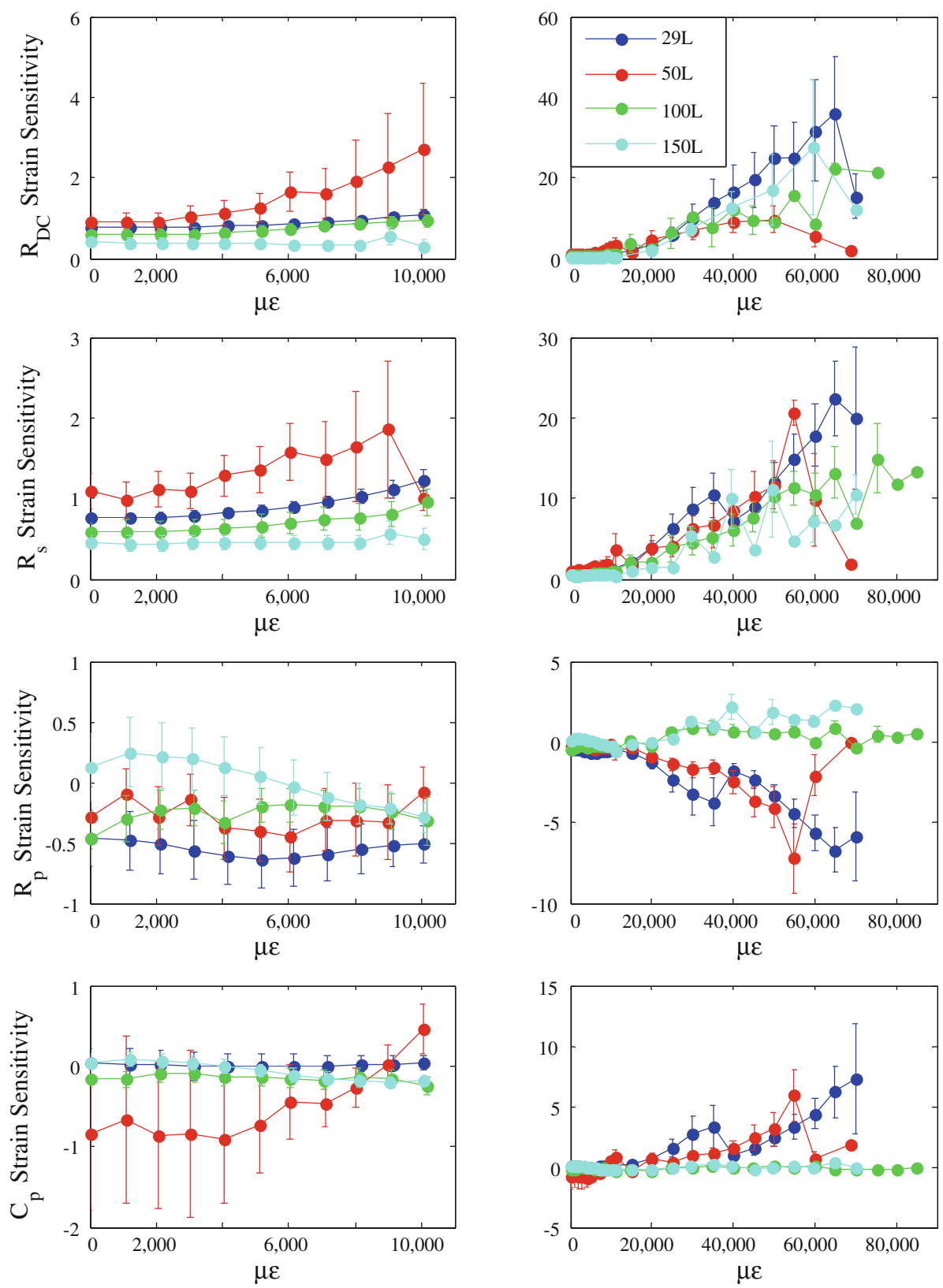

in the direction of the applied strain. This phenomenon has previously been modeled but only for cases of DC resistance measurements [58]. At higher strain values, the strain sensitivity between nanocomposites of different thicknesses becomes more distinct. In the case of $R_{\mathrm{p}}$, it exhibits an increasingly negative gage factor for films with 29 and 50 bilayers. These same thin films exhibit an increase in the gage factors for $C_{\mathrm{p}}$ within this region. We hypothesize that this behavior is due to Poisson's effect, which leads the glass fibers to exert compressive forces on the regions of the thin film contained among the fibers. These compressive forces decrease the inter-nanotube distances or apply pressure to the inter-nanotube connections, thereby reducing the resistance and increasing the capacitance. The increase in capacitance is justified by the fact that the value for capacitance is typically inversely related to the distance between conductive geometries.

However, the increasingly negative gage factors for (MWNT-PSS/PVA) 29 and (MWNT-PSS/PVA) 50 do not extend to films of 100 and 150 bilayers. After the lowstrain region, these nanocomposites respond to strain with a positive strain sensitivity for $R_{\mathrm{p}}$ and a near-zero strain sensitivity for $C_{\mathrm{p}}$, potentially due to the morphology of the thin film-fiber system of these thicker films. For these films, the number of deposited layers is presumably enough to completely fill-in the inter-fiber areas, and additional 
layers during film fabrication continue to deposit upon the pre-existing fiber bundle-thin film. As such, the volume of the thin film located within the fiber bundle would experience compressive forces due to Poisson's effect, whereas the volume of the thin film located outside the fiber bundle would be subjected only to longitudinal tensile forces.

\section{Summary and conclusions}

The purpose of this study is to characterize the strain sensing capabilities of piezoresistive carbon nanotubebased thin films deposited onto an anisotropic substrate such as glass fiber weaves. In this study, (MWNT-PSS/ PVA $)_{n}$ thin films of different thicknesses are deposited upon glass fiber substrates via an LbL deposition method. Each thin film specimen is loaded in uni-axial tension, and the load frame is commanded to hold its displacement and load at fixed strain intervals for electrical measurements. Time-domain DC resistance $\left(R_{\mathrm{DC}}\right)$ and EIS measurements are taken to probe the time- and frequency-domain thin film electromechanical response. For the EIS complex impedance measurements, an equivalent circuit model is also proposed for model fitting and to extract each circuit element's values corresponding to different levels of applied strain. From the time- and frequency-domain results, the measurements are able to capture two distinct thin film responses. First, the bulk resistive behavior of the film is characterized by the DC resistance measurements and the series resistor $\left(R_{\mathrm{S}}\right)$ of the EIS-fitted equivalent circuit model. Second, the inter-nanotube electrical behavior is captured by the equivalent circuit's parallel resistor $\left(R_{\mathrm{p}}\right)$ and parallel capacitor $\left(C_{\mathrm{p}}\right)$.

The nanocomposites' piezoresistive responses are wellcaptured by cubic smoothing spline fitting, and all the responses demonstrated two distinct sensitivities, depending on whether the film is strained at low $(0$ to $10000 \mu \varepsilon)$ or high strain $(>10000 \mu \varepsilon)$. Within the lower strain regime of less than $10000 \mu \varepsilon$, the bulk piezoresistivity exhibits a typical elastic response, while the inter-nanotube behavior is hypothesized to be due to carbon nanotube reorientation. In the higher strain regime, the bulk response is believed to suggest evidence for micro-cracking of the matrix and film. The inter-nanotube response, dependent on thin film thickness, exhibits a behavior that indicates that the thin film within the glass fiber bundles is subjected to compressive forces due to Poisson's effect, as evident from the negative and positive gage factors for $R_{\mathrm{p}}$ and $C_{\mathrm{p}}$, respectively. Further work will be done to fully understand the relationship between the piezoresistivity in the higher strain regime and micro-cracking within the composite structure. This outcome would further support this technology as being a viable in situ strain sensor for composite structures.

Acknowledgements The authors would like to thank Dr. Frank Yaghmaie, Ms. Yingjun "Irene" Zhao, Mr. Joe Fleck, and the Northern California Nanotechnology Center $\left(\mathrm{NC}^{2}\right)$ for assistance with acquiring the SEM images. The authors would also like to acknowledge the National Science Foundation (CAREER CMMI0642814) and the College of Engineering, University of California, Davis, for support of this research. Additional support has also been provided by the University of California Center for Information Technology Research in the Interest of Society (CITRIS).

Open Access This article is distributed under the terms of the Creative Commons Attribution Noncommercial License which permits any noncommercial use, distribution, and reproduction in any medium, provided the original author(s) and source are credited.

\section{References}

1. Roeseler B, Sarh B, Kismarton M, Quinlivan J, Sutter J, Roberts D (2009) Composite structures-the first 100 years. Composite design tutorial. Stanford University, Stanford

2. Bastianini F, Corradi M, Borri A, Tommaso A (2005) Construct Build Mater 19(7):525

3. Pantelides C, Alameddine F, Sardo T, Imbsen R (2004) J Bridge Eng 9(4):333

4. Guan H, Karbhari V, Sikorsky C (2007) J Intell Mater Syst Struct 18(8):809

5. Böger L, Wichmann M, Meyer L, Schulte K (2008) Compos Sci Technol 68(7-8):1886

6. Todo M, Nakamura T, Mada T, Takahashi K (1998) Adv Compos Mater 7(3):285

7. Buyukozturk O, Hearing B (1998) J Compos Construct 2(3):138

8. Quattlebaum J, Harries K, Petrou M (2005) J Bridge Eng 10(6):731

9. Rosenker MV (2006) Safety recommendation A-06-27 and -28. National Transportation Safety Board, Washington, DC

10. Gerardi T (1990) J Intell Mater Syst Struct 1(3):375

11. Staszewski W, Boller C, Tomlinson G (2004) Health monitoring of aerospace structures: smart sensor technologies and signal processing. Wiley, West Sussex

12. Bin L, Yaoyu L, Xin W, Zhongzhou Y (2009) In: Power electronics and machines in wind applications, 24-26 June 2009

13. Brownjohn J (2007) Philos Trans Roy Soc A: Math Phys Eng Sci 365(1851):589

14. Bhalla S, Yang Y, Zhao J, Soh C (2005) Tunnel Underground Space Technol Incorpor Trenchless Technol Res 20(5):487

15. Farrar C, Worden K (2007) Philos Trans Roy Soc A: Math Phys Eng Sci 365(1851):303

16. Lynch JP, Loh KJ (2006) Shock Vib Dig 38(2):91

17. Raghavan A, Cesnik C (2007) Shock Vib Dig 39(2):91

18. Van der Auweraer H, Peeters B (2003) Struct Health Monit 2(4):341

19. Chang F-K (2005) Structural health monitoring, 2005: advancements and challenges for implementation. DEStech Publications, Inc., Lancaster, PA

20. Halabe U, Vasudevan A, Klinkhachorn P, GangaRao H (2007) Nondestruct Test Eval 22(2):155

21. Qiu Z, Yao X, Yuan J, Soutis C (2006) Smart Mater Struct 15(4): 1047

22. Mirmiran A, Wei Y (2001) J Eng Mech 127(2):126 
23. Park H, Sohn H, Law K, Farrar C (2007) J Sound Vib 302(1-2): 50

24. Merzbacher C, Kersey A, Friebele E (1996) Smart Mater Struct 5(2): 196

25. Olin B, Meeker W (1996) Technometrics 38(2):95

26. Sweeting $T$ (1995) Int Stat Rev/Revue Internationale de Statistique 63(2):199

27. Iijima S (1991) Nature 354(6348):56

28. Saito R, Dresselhaus G, Dresselhaus M (1998) Physical properties of carbon nanotubes. Imperial College Press, London

29. Thostenson ET, Chou TW (2008) Nanotechnology. doi:10.1088/ 0957-4484/19/21/215713

30. Gao S, Zhuang RC, Zhang J, Liu JW, Mäder E (2010) Adv Funct Mater 20(12): 1885

31. Decher G (1997) Science 277(5330): 1232

32. Decher G, Schlenoff JB (2003) Multilayer thin films. WileyVCH, Weinheim, Germany

33. Loh KJ, Kim J, Lynch JP, Kam NWS, Kotov NA (2007) Smart Mater Struct 16(2):429

34. Loh KJ, Lynch JP, Shim BS, Kotov NA (2008) J Intell Mater Syst Struct 19(7):747

35. Gao L, Seliskar C (1998) Chem Mater 10(9):2481

36. Chen C, LaRue J, Nelson R (2009) Materials properties of polymer blends of poly (3, 4-ethlenedioxythiophene)/poly (styrenesulfonate)/N-methyl-2-pyrrolidinone (PEDOT: PSS: NMP) and polyvinyl alcohol (PVA). In: Cheng Z, Zhang Q, Bauer S, Wrobleski DA (eds) Polymer-based smart materials-processes, properties and application. Materials Research Society, Warendale, PA. doi: 10.1557/PROC-1134-BB05-41

37. ASTM Standard D3039 (2008) Standard test method for tensile properties of polymer matrix composite materials. ASTM International, West Conshohocken, PA. doi:10.1520/D3039_D3039M07, www.astm.org

38. Barsoukov E, Macdonald J (2005) Impedance spectroscopy: theory, experiment, and applications. Wiley-Interscience, Hoboken, NJ
39. Bauerle J (1969) J Phys Chem Solids 30(12):2657

40. Pham GT, Park YB, Wang S, Liang Z, Wang B, Zhang C, Funchess P, Kramer L (2008) Nanotechnology 19:325705

41. Spitalsky Z, Tsoukleri G, Tasis D, Krontiras C, Georga S, Galiotis C (2009) Nanotechnology 20:405702

42. Kang I, Schulz M, Kim J, Shanov V, Shi D (2006) Smart Mater Struct 15(3):737

43. Balberg I, Binenbaum N, Anderson C (1983) Phys Rev Lett 51(18): 1605

44. Essam J (1980) Rep Prog Phys 43(7):833

45. Kirkpatrick S (1973) Rev Mod Phys 45(4):574

46. Pike G, Seager C (1974) Phys Rev B 10(4):1421

47. Gao L, Thostenson ET, Zhang Z, Chou TW (2009) Carbon 47(5): 1381

48. Ogi K (2007) J Solid Mech Mater Eng 1(8):975

49. Gao L, Thostenson ET, Zhang Z, Chou T-W (2010) Adv Funct Mater 19(1):123

50. Vemuru S, Wahi R, Nagarajaiah S, Ajayan P (2009) J Strain Anal Eng Des 44(7):555

51. Wang S, Chung D (2007) J Mater Sci 42(13):4987. doi:10.1007/ s10853-006-0580-z

52. Wang X, Chung D (1998) Composites B 29(1):63

53. Wang X, Chung D (1998) Sens Actuators A 71(3):208

54. Hou T, Loh K, Lynch J (2007) Nanotechnology. doi:10.1088/ 0957-4484/18/31/315501

55. The Mathworks Inc. (2009b) MATLAB

56. NIST/SEMATECH e-Handbook of Statistical Methods (2010) http://www.itl.nist.gov/div898/handbook. Accessed 21 May 2010

57. Nofar M, Hoa S, Pugh M (2009) Compos Sci Technol 69(10): 1599

58. Taya M, Kim W, Ono K (1998) Mech Mater 28(1-4):53 